\title{
The Maternal Microbiota impact on Pregnancy Outcomes: A Systematic Review
}

\section{Poorva Rao ${ }^{1}$, Ketaki Rajwade ${ }^{1}$, Preeti Arora ${ }^{1}$, Anita Tiknaik ${ }^{1}$, Sreeja Parthasarthy ${ }^{1}$, Sharvari Ozalkar ${ }^{1}$, Pradnya Nikam ${ }^{1}$, Sanjay Gupte ${ }^{1,2 *}$ and Sarjan Shah ${ }^{1}$}

${ }^{1}$ Greenarray Genomic Research and Solutions, a division of Accurate Diagnostics

Pvt. Ltd., Kothrud, Pune, India

${ }^{2}$ Director, Gupte Hospital, Postgraduate Institution and Centre of Research in

Reproduction, Pune, India

*Corresponding Author: Sanjay Gupte, Director, Gupte Hospital, Postgraduate Institution and Centre of Research in Reproduction, Pune, India.
Received: December 13, 2021

Published: December 31, 2021

(C) All rights are reserved by Sanjay Gupte., et al.
Human Microbiome is the community of micro-organisms that lives in and on the human body. The human microbiome consist more than 100 trillion cells, which are ten times more than human cells and contain 27 times more genes than the human genome. The microbes reside on different sites of human body. For example, the skin, mouth, nasal cavity, gut, reproductive tract, and possibly the placenta host unique microbial communities [1]. Human microbiota includes archaea, Protista, bacteria, fungi, and viruses that exist on and within the human tissues, fluids and body cavities, along with varied anatomical sites. This microbiota plays an important role in immune system, human physiology and nutrition. In the human body, a large number of microorganisms exist in a mutualistic, peaceful relationship, but in immunosuppressed situations, a few of them become opportunistic, resulting to acute, deadly, and chronic illnesses. Every human body hosts unique microbiome within itself, which enables different individuals to behave differently when encountered with external stress [2]. From childbirth, a steady interaction (symbiosis) between the human body and its indigenous microbiota begins. Organisms that make up the microbiota have actively adjusted to their specific habitats and exist in their distinct niche within the human body through co-evolution. These creatures are identified as part of the body and cause a variety of alterations in lifetime. In reaction to host circumstances, the human microbiome is continually changing. At any one time, factors like age, nutrition, lifestyle, hormonal fluctuations, inherited genes, and underlying disease are key determinants of the human microbiome. However, a change in the human micro- biota makeup (dysbiosis) can result in life- threatening disorders [3]. A balanced microbiota has shown to play an important role in health sustenance [3]. The maternal pregnancy microbiome affects the mother's health and foetal development [4]. Thus, the dysbiosis of microbiome of pregnant woman may lead to a wide range of pregnancy problems. According to recent research, the number and composition of some gut microbiomes are altered during pregnancy complications. Maternal microbiomes have the potential to impact the physiological metabolism, immunological system, and inflammatory response of pregnant women [5]. The present review focuses and highlights the major impact of the maternal microbiomes in adverse pregnancy outcomes like, preterm birth, preeclampsia, gestational diabetes, and recurrent miscarriages.

\section{Preterm birth}

The rate of preterm delivery varies between $5 \%$ and $18 \%$ of all infants born in 184 countries. Premature births account for 3.5 million out of India's 27 million kids born each year [1]. While low socioeconomic status is a risk factor for preterm birth. Also, the mother's age (under 20 or $>35$ years), sexually transmitted infections, underweight, obesity, chronic health conditions, short inter pregnancy intervals, and tobacco and substance use, account for preterm birth [6].

Preterm labour is currently known to be caused by a variety of factors, including infection or inflammation, uteroplacental ischaemia or haemorrhage, uterine over distension, stress, and other 
immune-mediated processes [7]. In the second and third trimesters, maternal abdominal surgery might increase contractions, resulting in a premature birth. Medical conditions in the mother, including thyroid illness, asthma, diabetes, and hypertension, are linked to a higher likelihood of premature births, many of which are due to maternal complications. A history of cervical cone biopsy samples or loop electrocautery excision treatments for premalignant cervical diseases, as well as numerous uterine defects such the existence of a septum, have all been linked to an increased risk of spontaneous premature birth [8].

Intrauterine infection is one of the reasons for preterm birth, which is mostly caused by oral microbial species than the urogenital tract microbiome. From study, which found out the presence of gut-associated microbes in amniotic fluid of women with preterm premature rupture of membranes, it can be hypothesised that, the gut microbiome is a potential source of intrauterine infection. Gut-associated bacteria might colonise the vaginal canal and ascend also, hematogenous transmission from the gut lumen to the circulation may occur [6]. The vaginal microbiota associated with bacterial vaginosis has been linked to an increased risk of sexually transmitted infections, which can lead to premature delivery. Bacterial vaginosis is also a risk factor for preterm delivery, yet a comprehensive, detailed analysis indicated that even eradicating bacterial vaginosis did not result in a substantial reduction in preterm birth risk [6].

\section{Gestational diabetes}

Gestational diabetes raises the risk of maternal problems such gestational hypertension, which raises the chance of Caesarean delivery and premature birth. Pregnant women's gut microbiomes have been linked to pre-pregnancy body weight and excessive weight gain during pregnancy. A recent research that looked at the gut microbiota's composition during pregnancy discovered considerable gut microbiome remodelling from the first to the third trimester [5]. The composition of the first trimester was most similar to that of non-pregnant women, whereas the changes from the first to the third trimester included increased between- subject diversity but decreased within-subject diversity, with the majority of women demonstrating increased abundance of Proteobacteria, a microorganism associated with inflammation-related shifts in the gut microbiome. During the first trimester, women who acquired gestational diabetes had the least diverse gut microbiota [6].
Ruminococcaceae, Parabacteroides distasonis, and Prevotella microbiota were shown to be abundant in women with gestational diabetes in several investigations. These bacterial communities are linked to glucose metabolism and insulin signalling. Furthermore, increased expression of serum zonulin, a measure of gut epithelial permeability, in women with gestational diabetes during early pregnancy suggests a potential relationship between gut microbiota and gestational diabetes. But, the relationship between gut microbiota dysbiosis and metabolism in women with gestational diabetes is still unclear. Lifestyle changes and metformin medication revealed signs of gut microbiota manipulation and can be shown to be beneficial in maintaining glucose homeostasis [10].

\section{Preeclampsia}

Pregnancies complicated by severe preeclampsia have a greater risk of maternal and perinatal morbidity, as well as a higher rate of placental abruption. Preeclampsia also puts the offspring at danger later in life, with a two-fold increased risk of stroke. The "foetal roots of adult illness theory" and shared genetic risk factors between mother and foetus have previously been used to explain this increased risk. However, a new theory proposes that preeclampsia- related changes in the maternal gut microbiota may affect embryonic gut development and contribute to future illness [11]. One research looked at the presence of bacteria in preeclamptic women's amniotic fluid and found that the amniotic fluid infection has a little impact in the development of preeclampsia [10].

A study has detected the presence of Lactobacillus iners, Ureaplasma, Sneathia, Leptotrichia, and Streptococcus species in the amniotic fluid of women with pre-eclampsia, which used techniques like, traditional microbial culture techniques and broad range and group-specific polymerase chain reaction (PCR) assays targeting the $16 \mathrm{~S}$ ribosomal RNA (rRNA) gene. Another study found out the the presence of periodontal pathogens such as Actinobacillus actinomycetemcomitans, Fusobacterium nucleatum, Porphyromonas gingivalis, Prevotella intermedia, Tannerella forsythensis, and Treponema denticola, which used real-time PCR to investigate placental tissue. But, the particular organism(s) that might cause preeclampsia remains unknown. The current investigation was founded on the idea that one or more infectious pathogens present in the placenta induce pre-eclampsia, with a dominant organism causing the illness $[9,11]$. 


\section{Recurrent miscarriage}

A study, conducted on a group of women suffered through the $\mathrm{RM}$, showed increase in species richness and considerable shift in total microbial diversity of vaginal microbiome. These findings showed that abnormalities in the vaginal microbiome profile in individuals with recurrent miscarriage might be a cause or a consequence of the altered vaginal microbiome composition [12]. There are presently, no studies that show a direct link between recurrent miscarriages and vaginal microbiome. The vaginal flora is less diverse than the intestinal flora, with women of reproductive age having up to 40 species of bacteria and facultative anaerobic bacteria, such as Lactobacillus, Bacteroides, Coccidioides, Corynebacterium, Escherichia coli, Velveti, and Gardneria [12,13]. Bacterial vaginosis is defined by the absence of lactobacilli and a rise in Gram-variable and Gram-negative rods, with Gardnerella vaginalis, Bacteroidetes, Prevotella, and Mobiluncus species being the most common. Early in pregnancy, an aberrant vaginal microbiota is a known risk factor for premature delivery and low birth weight [14].

\section{Bibliography}

1. Dunlop AL., et al. "Maternal Microbiome and Pregnancy Outcomes That Impact Infant Health: A Review". Advances in Neonatal Care 15.6 (2015): 377-385.

2. Gupta P., et al. "Diversity of Vaginal Microbiome in Pregnancy: Deciphering the Obscurity". Frontiers in Public Health 8 (2020): 326.

3. Ogunrinola GA., et al. "The Human Microbiome and Its Impacts on Health". International Journal of Microbiology 2020 (2020): 8045646.

4. Turjeman Sondra., et al. "The gut microbiome in pregnancy and pregnancy complications". Current Opinion in Endocrine and Metabolic Research 18 (2021).

5. Yao Y., et al. "The Role of Microbiomes in Pregnant Women and Offspring: Research Progress of Recent Years". Frontiers in Pharmacology 11 (2020): 643.

6. Hamilton BE., et al. "Births: preliminary data for 2011". $N a$ tional Vital Statistics Reports 61.5 (2012): 1-18.

7. Goldenberg RL., et al. "Epidemiology and causes of preterm birth”. Lancet 371.9606 (2008): 75-84.
8. Krupa FG., et al. "Predictors of preterm birth". International Journal of Gynecology and Obstetrics 94.1 (2006): 5-11.

9. Santacruz A., et al. "Gut microbiota composition is associated with body weight, weight gain and biochemical parameters in pregnant women". British Journal of Nutrition 104.1 (2010): 83-92.

10. Zhang F., et al. "Alteration of vaginal microbiota in patients with unexplained recurrent miscarriage". Experimental and Therapeutic Medicine 17.5 (2019): 3307-3316.

11. Amarasekara R., et al. "Microbiome of the placenta in preeclampsia supports the role of bacteria in the multifactorial cause of pre-eclampsia". Journal of Obstetrics and Gynaecology Research 41.5 (2015): 662-669.

12. Wells CL., et al. "Role of anaerobic flora in the translocation of aerobic and facultatively anaerobic intestinal bacteria". Infection and Immunity 55.11 (1987): 2689-2694.

13. Hasain Z., et al. "Gut Microbiota and Gestational Diabetes Mellitus: A Review of Host-Gut Microbiota Interactions and Their Therapeutic Potential". Frontiers in Cellular and Infection Microbiology 10 (2020): 188.

14. Petricevic L., et al. "Characterisation of the vaginal Lactobacillus microbiota associated with preterm delivery". Scientific Report 4 (2014): 5136.

\section{Assets from publication with us}

- Prompt Acknowledgement after receiving the article

- Thorough Double blinded peer review

- Rapid Publication

- Issue of Publication Certificate

- High visibility of your Published work

Website: www.actascientific.com/

Submit Article: www.actascientific.com/submission.php

Email us: editor@actascientific.com

Contact us: +919182824667 